Croatian Journal of Philosophy

Vol. XXI, No. 63, 2021

https://doi.org/10.52685/cjp.21.63.4

Received: March 16, 2021

Accepted: December 1, 2021

\title{
On Formalizing Logical Modalities
}

\author{
LUIGI PAVONE \\ University of Palermo, Palermo, Italy
}

This paper is in the scope of the philosophy of modal logic; more precisely, it concerns the semantics of modal logic, when the modal elements are interpreted as logical modalities. Most authors have thought that the logic for logical modality - that is, the one to be used to formalize the notion of logical truth (and other related notions)—is to be found among logical systems in which modalities are allowed to be iterated. This has raised the problem of the adequacy, to that formalization purpose, of some modal schemes, such as $\mathbf{S}_{4}$ and $\mathbf{S}_{5}$. It has been argued that the acceptance of $\mathbf{S}_{5}$ leads to non-normal modal systems, in which the uniform substitution rule fails. The thesis supported in this paper is that such a failure is rather to be attributed to what will be called "Condition of internalization." If this is correct, there seems to be no normal modal logic system capable of formalizing logical modality, even when $S_{5}$ is rejected in favor of a weaker system such as $S_{4}$, as recently proposed by McKeon.

Key words: Logical truths; logical formality; uniform substitution; Kripke semantics; Carnap-style semantics.

\section{Introduction}

Kripke (1959, 1963) defined general validity for extending the notion of a valid formula to intensional languages, providing a modal semantics that has proved very useful in clarifying a wide range of modal notions. This paper addresses the problems that arise under a special interpretation of modal operators in terms of logical modalities, i.e., reading " $L$ " as "it is logically necessary that...", and " $M$ " as 'it is logically possible that...' To begin with, can Kripkean general validity capture the meaning of a valid formula in a first-order modal language when modal operators are interpreted in such a way? A line of thought, which can be traced back to Pollock (1966), says it cannot. This view invokes 
an alleged intuitive truth about logical possibility-let us call it "Necessary Logical Possibility" (NLP) - according to which true sentences about what is logically possible are logically true. For example, consider the following logically possible sentence, (i) "Jones passes the exam". Since (ii) "possibly, Jones passes the exam", obtained from (i) by prefixing the logical possibility adverb "possibly", is true, NLP states that (ii) is logically true as well. Let us call "logical possibility sentences" all true sentences about the logical possibility of states of affairs.

If NLP is right, there are intuitive modal logical truths, logical possibility sentences, such as (ii), that do not fall under the Kripkean concept of general validity. The point against Kripke semantics is that the purely formal (or logical) sense of modal operators is not captured if they are allowed to range over arbitrary non-empty sets of (logically) possible worlds. As Cocchiarella (1975) puts it, such a construction of modal operators,

by allowing the exclusion of some of the worlds (models) of a logical space, imports material conditions into the semantics of modal operators. This exclusion, however appropriate for the representation of non-logical [...] modalities, is quite inappropriate for the representation of what are purported to be merely formal or logical modalities. (1975: 13)

The aim of construing an alternative semantics for logical modality was pursued in different (but convergent) ways in Cocchiarella (1975) and Hanson and Hawthorne (1985), by using a fixed collection of logically possible worlds. As we will see in detail in Section 3, their proposal can be thought of as a version of Carnap's modal logic (1947) based on extensional models. Let us call it "Carnap-style semantics."

The main problem with this alternative semantics raises on the basis of what can be called the Quinean sense of logical formality, on which logical formality is closely related to the principle of Uniform Substitution (US): "a logical truth [...] is definable as a sentence from which we get only truths when we substitute sentences for its simple sentences" (Quine 1970: 50). As noticed by Makinson (1966), and more recently by McKeon (2005), US fails in Carnap-style semantics, in which the following puzzling situation arises: the notion of a valid formula respects the intuitions of NLP, but at the cost of abandoning the formality of logic conceived in terms of substitutivity.

Section 2 shows why Kripke semantics should be considered inadequate to represent logical modality. Section 3 shows how proponents of Carnap-style semantics propose to solve the problems affecting Kripke semantics. Section 4 shows that Carnap-style semantics is not without problems in its own right, since it violates US, thereby undermining the notion of logical formality understood in terms of substitutivity.

McKeon (2005) has argued that the main reason for the failure of US is the acceptance of the modal scheme $\mathbf{S}_{5}$, i.e. " $M \varphi \rightarrow L M \varphi$ ", as a valid logical scheme in formalizing logical modality. In defense of Kripke semantics, the author maintains that the correct logic for logical modal- 
ity must be a system that is "at least as strong as $S_{4}$, but not as strong as $S_{5}$ " (2005: 313). In contrast, I will argue that substitutivity fails due to a more general desideratum for modal systems that aim to represent logical modality-what I will call "Condition of Internalization" (CI). If this is correct, McKeon's proposal to preserve US within the framework of Kripke semantics fails, due to the normality of the formalization he proposes, where by "normality" it is meant the property of being a logic in which the propositional tautologies, the rule of Necessitation $(\mathrm{N})$, i.e. if $\varphi$ is logically true so is $L \varphi$, and the Distribution axiom, i.e. $L(\varphi \rightarrow \psi)$ $\rightarrow(L \varphi \rightarrow L \psi)$, hold. Section 4.1 considers Schurz's proposal (2001) to define logical formality in terms of "semantically isomorphic substitutions". I will argue that such a definition is circular. Finally, in Section 4.2 I will argue against a further argument proposed by McKeon in favor of Kripke semantics, what I will call "the argument from analogy". That argument appears to be flawed if one takes into account all the implications of CI when it is applied to the property of being contextinsensitive that is unanimously attributed to all valid first-order formulas.

\section{Kripke semantics}

Let $\mathfrak{L}$ be a first-order language without individual constants. $\mathfrak{L}$ is the same as that for the standard first-order logic (with the symbol of identity) but supplemented with the modal letter " $L$ " and the corresponding formation rule: if $\varphi$ is a formula, so is $L \varphi$. One can add the modal letter " $M$ " for the notion of possibility, by definition: $M \varphi$ means $\neg L \neg \varphi$. The further details are assumed to be defined. Kripke possible worlds semantics can be introduced as follows.

Let $w=\left\langle d_{0}, d_{i}, V\right\rangle$ be an outer/inner sub-model such that $d_{0}$ is a possible empty outer domain, and $d_{i}$ is a non-empty inner domain. ${ }^{1}$ These two sets are disjoint and their union counts as the domain of discourse of $w$, that is $d(w) . V$ is an interpretation function assigning extensions to the predicate letters of $\mathcal{L}$, so that for any $n$-place predicate letter $\boldsymbol{P}$, $V(\boldsymbol{P}) \subseteq d(w)^{n}$.

Let $\varphi$ be an atomic formula $\boldsymbol{P}^{n}\left(\boldsymbol{x}_{1} \ldots \boldsymbol{x}_{n}\right)$, and $v$ an assignment from individual variables into $d(w)$, we say that $v$ satisfies $[w]$ (satisfies at $w) \varphi$ iff $\left\langle v\left(\boldsymbol{x}_{1}\right), \ldots, v\left(\boldsymbol{x}_{n}\right)\right\rangle \in V(\boldsymbol{P})$. If $\varphi$ has the form $\boldsymbol{x}_{1}=\boldsymbol{x}_{2}, v$ satisfies $[w]$ $\varphi$ iff $v\left(\boldsymbol{x}_{1}\right)=v\left(\boldsymbol{x}_{2}\right)$. If $\varphi$ has the form $\neg \psi, v$ satisfies [w] $\varphi$ iff $v$ does not satisfies $[w] \psi$. If $\varphi$ has the form $\psi \vee \gamma, v$ satisfies [w] $\varphi$ iff $v$ satisfies $[w]$ $\psi$ or $v$ satisfies $[w] \gamma$. First-order quantifiers are restricted to inner domains, so that $v$ satisfies $[w] \forall \boldsymbol{x} \psi(\boldsymbol{x})$ iff for every $v^{\prime}$ different from $v$ at most for $\boldsymbol{x}$ such that $v^{\prime}(\boldsymbol{x}) \in d_{i}, v^{\prime}$ satisfies $[w] \psi(\boldsymbol{x})$.

A Kripke model is a four-tuple $\Re=\langle W$, @, $R, D\rangle$, where $W$ is an arbitrary non-empty set of sub-models, @ is a member of $W$ representing

${ }^{1}$ Intuitively, $d_{i}$ is the set of all objects existing in $w$, and $d_{0}$ is the set of all merely possible object with respect to $w$. 
the actual state of affairs, $R$ is the customary accessibility relation defined on $W$. For any $w \in W, d(w)=D$. Note that $W$ is allowed to vary from model to model: if $\mathbf{W}$ is the set of all possible worlds, $W \subseteq \mathbf{W}$. A semantically autonomous subset of Kripke models—called "constant domains semantics"-consists of those models $\langle W, @, R, D\rangle$ such that for every sub-model $w \in W, d_{i}(w)=D$.

The notion of general validity can be defined in terms of truth[भ] (truth in $\Re$ ) in a number of steps. Let $\varphi$ be an atomic formula, and $v$ an assignment from individual variables into $D$, we say that $v$ satisfies[or] (satisfies in $\mathscr{\text { RC }} \varphi$ at a world $w \in W$ iff $v$ satisfies $[w] \varphi$. If $\varphi$ has the form

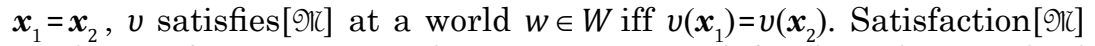
conditions for propositional connectives are defined in the standard way. First-order quantifiers are restricted to inner domains, so that $v$ satisfies[भ] $\forall \boldsymbol{x} \psi(\boldsymbol{x})$ at a world $w \in W$ iff for every $v^{\prime}$ different from $v$ at most for $\boldsymbol{x}$ such that $v^{\prime}(\boldsymbol{x}) \in d_{i}(w), v^{\prime}$ satisfies[भ] $\psi(\boldsymbol{x})$ at $w$. Modal operators are semantically defined as follows: $v$ satisfies[0r] $L \varphi$ at a world $w \in W$ iff $v$ satisfies[९] $\varphi$ at every worlds $w^{\prime} \in W$ such that $(w$, $\left.w^{\prime}\right) \in R$. A formula $\varphi$ is said to be true[थ] at a world $w \in W$ iff for any $v, v$ satisfies[ๆ] $\varphi$ at $w . \varphi$ is said to be true[Ю] iff $\varphi$ is true[भ] at @. Finally, a formula $\varphi$ is said to be generally valid iff $\varphi$ is true[Юr] for any model or.

If the accessibility relation $R$ is reflexive, transitive, and symmetrical, $\mathbf{S}_{5}$ turns to be generally valid. Under the logical interpretation of the modal operators, $\mathbf{S}_{5}$ formalizes NLP. Indeed, under that interpretation, that scheme says: if a sentence $\varphi$ is logically possible, then it is logically necessary that it is so. As pointed out by McKeon, the acceptance of NLP entails the acceptance of the validity of $\mathbf{S}_{5}$ (cf. 2005: 308). Therefore, those who accept NLP will be committed to formalize the notion of logical modality within modal systems in which $\mathbf{S}_{5}$ is valid. Such a correspondence between NLP and $\mathbf{S}_{5}$, due to the fact that $\mathbf{S}_{5}$ is the formalization in the object language of the meta-theorem NLP, is just a case of a more general desideratum for modal systems that aim to represent logical modality-let us call it "Condition of Internalization" (CI). CI can be stated as follows: whenever $\Gamma$ is a sentence in the meta-language of a logical system and $\varphi$ is the formalization of it in the object-language of that system, $\Gamma$ is a meta-theorem of the system iff $\varphi$ is valid. For example, if $\Gamma$ is the meta-theorem (of a logical modal system) that all true sentences are logically possible, the scheme $\varphi \rightarrow M \varphi$, which formalizes $\Gamma$ in the object-language (of that system) under the logical reading of the modal operators, must be valid; if it was not, the following puzzling situation would arise: there would be a model $\Re$ such that some sentence of the form $\varphi \rightarrow M \varphi$ would not be true[গ], in contrast with $\Gamma$, which states that it is not logically possible that something true is not logically possible.

The main problem pointed out by the critics of Kripke semantics is that it is unable to account for the intuitive truth expressed by NLP. 
Although $\mathbf{S}_{5}$, qua formalization of NLP, can be assumed as generally valid (with the appropriate conditions on the accessibility relation), yet NLP cannot be a meta-theorem of the system $S_{5}$. In fact, NLP requires that all sentences of the form $M \varphi$ be logically true, for any consistent first-order sentence $\varphi$, but that is not the case for consistent first-order sentences that are not valid in first-order logic. Let $\varphi$ be a first-order sentence of that sort, for example (i) again. Since there is a sub-model $w$ such that the contradictory of (i), i.e. (iii) "Jones does not pass the exam", is true at $w$-the existence of such a sub-model is guaranteed by the fact that (i) is not of a valid first-order logical form -, a Kripke model $\Re=\langle W$, @, $R, V\rangle$ can be formed out of $w$ in such a way that $W=\{@\}$, and @=w. Given that (iii) is true at @, and @ is the only possible world in $W$, the corresponding modal sentence (ii), of the form $M \varphi$, will be false in $\Re$, contrary to what NLP states: that all logical possibility sentences are logically true. In contrast to NLP, there are counter Kripke models for $M \varphi$, for any consistent but not valid first-order sentence $\varphi$. In other terms, the constraint CI turns out to be violated with respect to the relationship between NLP and $\mathbf{S}_{5}$.

The Kripkean notion of general validity turns out to be undermined by some others difficulties identified by Pollock (1966). According to Pollock, Kripke semantics does not capture a fundamental feature of logical truths, i.e. the fact that they are not context-sensitive: logical truths should be true regardless of their domain of discourse. ${ }^{2}$ For example, sentences of the form $\exists \boldsymbol{x} \psi(\boldsymbol{x}) \wedge \exists \boldsymbol{x} \neg \psi(\boldsymbol{x})$ should not count as logical truths, because they are true only if the intended domain of discourse contains more than one object. Those sentences are contextsensitive, because they are domain-sensitive. Now, if " $L$ " means "it is logically true that...", the scheme $\neg L(\exists \boldsymbol{x} \psi(\boldsymbol{x}) \wedge \exists \boldsymbol{x} \neg \psi(\boldsymbol{x})$ formalizes in the object language the meta-theorem that sentences of the form $\exists \boldsymbol{x} \psi(\boldsymbol{x}) \wedge \exists \boldsymbol{x} \neg \psi(\boldsymbol{x})$ are not logically true. Therefore, it should be valid, and the scheme $L \neg L(\exists \boldsymbol{x} \psi(\boldsymbol{x}) \wedge \exists \boldsymbol{x} \neg \psi(\boldsymbol{x}))$ as well in virtue of the axiom $\mathbf{S}_{5}$, but this is not the case in Kripke semantics (cf. 1966: 316). Again, the condition CI is not obeyed.

\section{Carnap-style semantics}

In formalizing logical modalities, those who accept NLP are committed to a modal logic system in which $\mathbf{S}_{5}$ is valid and NLP is a meta-theorem of that system, i.e. a logic in which all true sentences of the form $M \varphi$ are logically true. This aim was pursued in the framework of Carnap's modal logic (1947) by using a fixed space of all logically possible worlds.

Carnap's modal language had an infinite collection of individual constants. The notion of logical necessity, relative to an appropriate domain of discourse, was defined as truth in every state-description,

${ }^{2}$ Indeed, the domain of discourse of a sentence can be considered as a part of a larger context that also includes other elements, such as the speaker, time, etc. 
where a state-description is a set $S$ such that for any atomic sentence $\varphi$, just one of $\varphi$ or $\neg \varphi$ is in $S$. $\varphi$ holds at $S$ iff $\varphi \in S$. Given an assignment function $v$ from individual variables into intensions, that is, assignments "of exactly one individual constant to each state-description" (Carnap 1947: 181), Carnapian satisfaction conditions can be defined as follows. If $\varphi$ is an atomic formula $\boldsymbol{P}^{n}\left(\boldsymbol{x}_{1} \ldots \boldsymbol{x}_{n}\right), v$ satisfies $[S]$ (satisfies at $S) \varphi$ iff $v\left(\boldsymbol{x}_{1}\right)=\boldsymbol{i}_{i}$, where $\boldsymbol{i}_{i}$ is an intension, $\boldsymbol{i}_{i}(S)=\boldsymbol{a}_{i}$, and the sentence obtained from $\varphi$ by appropriate substitutions, i.e. $\boldsymbol{P}^{n}\left(\boldsymbol{a}_{1} \ldots \boldsymbol{a}_{n}\right)$, holds at $S$. If $\varphi$ has the form $\boldsymbol{x}_{1}=\boldsymbol{x}_{2}, v$ satisfies $[S] \varphi$ iff $v\left(\boldsymbol{x}_{1}\right)=\boldsymbol{i}_{1}, v\left(\boldsymbol{x}_{2}\right)=\boldsymbol{i}_{2}$, and $\boldsymbol{i}_{1}(S)=\boldsymbol{i}_{2}(S)$. If $\varphi$ has the form $\neg \psi, v$ satisfies $[S] \varphi$ iff $v$ does not satisfies $[S]$ $\psi$. If $\varphi$ has the form $\psi \vee \gamma, v$ satisfies $[S] \varphi$ iff $v$ satisfies $[S] \psi$ or $v$ satisfies $[S]$ $\gamma$. If $\varphi$ has the form $\forall \boldsymbol{x} \psi(\boldsymbol{x}), v$ satisfies $[S] \varphi$ iff for any $v^{\prime}$ different from $v$ at most for $\boldsymbol{x}, v^{\prime}$ satisfies $[S] \psi(\boldsymbol{x})$. Finally, if $\varphi$ has the form $L \psi, v$ satisfies $[S] \varphi$ iff $v$ satisfies $\left[S^{\prime}\right]$ for every state-description $S^{\prime}$.

Carnap's modal logic validates both $\mathbf{S}_{4}$ and $\boldsymbol{S}_{5}$. NLP is ensured by the uniqueness of the logical space of possibilities. If $\varphi$ is a satisfiable sentence whatsoever, then there is a state-description $S$ such that $\varphi$ holds at $S$. Since $S$ is in the single fixed logical space of possibility, $M \varphi$ will hold at all state-descriptions.

This approach to validity for modal languages was adopted using extensional models, instead of state-descriptions, by Montague (1974), Beth (1960), and more recently by Cocchiarella (1975) and Hanson and Hawthorne (1985). A simple way to state the Carnapian notion of validity in terms of extensional models_call it C-validity-is to use our language $\mathfrak{L}$ and constant domains semantics. One can state that a Carnap-style model is a constant domains model $\langle\mathbf{W}$, @, $R, D\rangle$ such that $R$ is reflexive, transitive, symmetrical, and $\mathbf{W}=\left\{w: d_{i}(w)=D\right\}$, i.e. the set of all sub-models of the same domain of discourse. Following Carnap, the number of the objects of the domain of discourse can be infinite. Thus a sentence will be valid iff it is true in all models of the same logical space of possibility. ${ }^{3}$ This time, the sentence (ii) turns out to be logically true, for it instantiates a $\mathrm{C}$-valid formula, in accordance with the desiderata of NLP.

\section{Formality of logics and the condition of substitutivity}

Carnap-style modal semantics ensures NLP and the validity of $\mathbf{S}_{5}$ by using a single logical space including all logical possibilities, but at the cost of abandoning Quine's sense of the formality of logic, according to

${ }^{3}$ An alternative extensional version of the Carnapian notion of validity-call it C-validity* — can be defined by stating that a formula is C-valid* iff it is true in all models for any domain of discourse. However, this choice is open to some problems, because not all logical possibility sentences are C-valid*. All logical possibility sentences of the form $M \exists \boldsymbol{x} \exists \boldsymbol{y}(\boldsymbol{x} \neq \boldsymbol{y})$ are not C-valid*, although $\exists \boldsymbol{x} \exists \boldsymbol{y}(\boldsymbol{x} \neq \boldsymbol{y})$ is a logically satisfiable scheme. On the other hand, those of the form $M \exists \boldsymbol{x} \forall \boldsymbol{y}(\boldsymbol{x}=\boldsymbol{y})$ are C-valid*, but not C-valid. Thus NLP would hold in general for all logical possibility sentences whose truth conditions do not require constraints on the domains of discourse in addition to that of being non-empty. 
which logical formality is closely related to US: if $\varphi$ is valid, so is $S(\varphi)$, where $s(\varphi)$ is obtained from $\varphi$ by a substitution function $s$ from predicative letters into formulas of the object-language. The weak point of this sort of modal semantics is that the class of its valid formulas is not closed under US. Let $\varphi$ be a sentence of the form $M \psi$, where $\psi$ is an arbitrary atomic sentence. $\varphi$ is C-valid in virtue of the Carnap-style semantic rules, but if $s$ is a substitution function such that $s(\psi)=\psi \wedge$ $\neg \psi, s(\varphi)$ will be obviously false, and even logically false. Why does substitution fail in Carnap-style modal semantics?

According to Makinson (1966), this is because "schematism" is neglected when modal formulas are understood in terms of what the author calls "naive modal rules", which are exemplified by Carnap's modal semantics. By schematism he means the thesis that a logical scheme is acceptable iff all its instantiations by uniform substitutions are logically true in terms of the naive modal rules (cf. 1966: 334). Based on this, Makinson argued that the scheme $M \varphi$ is not acceptable, because some of its instantiations, e.g. those of the form $M(\varphi \wedge \neg \varphi)$, are not logically true. However, in Makinson's proposal, US preserves the property of being acceptable (referred to logical schemes), but not the property of being logically true referred to sentences, thereby leaving open the possibility to get false sentences from logical truths by uniform substitution. This amounts to rejecting Quine's understanding of logical formality in terms of substitutivity. In fact, consider the true sentence (ii), of the form $M \varphi$. Following Makinson, (ii) is logically true, in virtue of the naive modal rules, although the modal logical scheme from which it is formed out is not acceptable. This begs the question: in what sense should the truth of (ii) be a matter of form or structure? There seems to be no straightforward answer in Makinson's combination of schematism and naive modal rules.

The failure of US is ascribed to the scheme $\mathbf{S}_{5}$ by McKeon (2005). For the safety of substitutivity, McKeon proposed to reject NLP, which obliges us to accept the validity of $\mathbf{S}_{5}$. According to McKeon, the correct formalization of the logic of logical modality is a modal system that is "at least as strong as $S_{4}$, but not as strong as $S_{5}$ ". The acceptance of the validity of $\mathbf{S}_{4}$ can be motivated by some considerations. First, once iteration is admitted, $\mathbf{S}_{4}$ seems to be more intuitive than $\mathbf{S}_{5}$. Unlike $\mathbf{S}_{5}, \mathbf{S}_{4}$ does not violate US. Moreover, $\mathbf{S}_{4}$ formalizes N, which characterizes all normal modal systems. In fact, under the logical reading of the modal operators, $\mathbf{S}_{4}$ says that if a sentence is logically true, it is logically true that it is so. On the grounds of $\mathrm{CI}$, it is not possible to accept $\mathrm{N}$ without accepting the validity of $\mathbf{S}_{4}$, just as we cannot accept NLP without accepting the validity of $\mathbf{S}_{5}$. Since $\mathrm{N}$ is a meta-theorem of normal modal logics, and $\mathbf{S}_{4}$ formalizes it (under the logical reading of the modal operators) in their object-language, the choice of a system that is "at least as strong as $S_{4}$ ", as suggested by McKeon, is needed if one wishes to formalize logical modality in a normal modal system. 
My thesis is that McKeon's defense of Kripke semantics, whose aim is to preserve US, cannot work. In fact, the modal logical system proposed by McKeon would require a semantics validating the formalization in the object-language not only of N, i.e. $\mathbf{S}_{4}$, but also of US, in accordance with CI. The correct logic for logical modality should be such that the following principle holds: if $L \varphi$ is true, so is $L s(\varphi)$, where $s(\varphi)$ is an instantiation of $\varphi$ by uniform substitution. Accordingly, the following logical modal scheme should be valid: $L \varphi \rightarrow L s(\varphi)$. Let us call it "US", which is the schematic internalization of US in the object-language. Under the logical reading of its modal elements, US says that if $\varphi$ is logically true, so is any instantiation of it by US. That lands us with a version of the same old problem: US will be violated in Kripke semantics for any hypothetical normal modal logic in which its schematic internalization is valid.

Let $\varphi$ be an interpreted formula of the form $M \psi$, where $\psi$ is atomic. Two possible cases can be hypothetically considered: when $\varphi$ is logically true, when it is not. If $\varphi$ is logically true, then all instantiations of it by US will be logically true, but this contrasts to the evidence that some of them, those of the form $M(\psi \wedge \neg \psi)$, are logically false. On the other hand, if $\varphi$ is not logically true, then the contradictory of it, of the form $L \neg \psi$, will be (at least) logically possible, i.e. there must be a logically possible state of affairs at which $L \neg \psi$ is true. Therefore, it must be the case that for some Kripke model $\Re=\langle W, @, R, D\rangle, \psi$ fails to be true in every possible $w \in W$, from which the truth[ब] of $L \neg \psi$ is obtained. Under the logical reading of modal operators, that $L \neg \psi$ is true[๑r] means that it is true[बr] that $\neg \psi$ is logically true. By the following instantiation of US, i.e. $L \neg \psi \rightarrow L s(\neg \psi)$, and modus ponens, it must also be the case that for any substitution function $s, L s(\neg \psi)$ be true[बr], for some $\pi$. This means that if $s(\neg \psi)=\neg(\psi \vee \neg \psi)$, then $L \neg(\psi \vee \neg \psi)$ must be true[भ], for some $\Re$. Since the scheme $\psi \vee \neg \psi$ is a propositional tautology, there is no logical possible state of affairs at which $\psi \vee \neg \psi$ will fail to be true, hence it is not possible that $\neg(\psi \vee \neg \psi)$ is logically true, and so there is no Kripke model or such that $L \neg(\psi \vee \neg \psi)$ is true[ब]. Such a contradictory circumstance can be described as follows: in the logic of logical modality, one will inconsistently get both $M L \neg(\psi$ $\vee \neg \psi)$, namely it is logically possible that $\neg(\psi \vee \neg \psi)$ is logically true, and $\neg M L \neg(\psi \vee \neg \psi)$, namely it is not logically possible that $\neg(\psi \vee \neg \psi)$ is logically true.

This suggests that US fails in every normal modal logic in which its internalization US is assumed to be valid in accordance with CI. If this is right, McKeon's proposal to combine US with a normal modal system "at least as strong as $S_{4}$, but not as strong as $S_{5}$ " fails because of the normality of the modal system proposed. 


\subsection{Semantically isomorphic substitutions}

According to Schurz (2001), Carnap's logic is "the only complete modal logic" if modal operators express logical modalities (2001: 365). He addressed the problem of logical formality with the C-valid scheme $M \varphi$ (with $\varphi$ logical consistent) by redefining the notion of logical formality in terms of what the author call "semantically isomorphic substitutivity".

He proposes to distinguish between semantically isomorphic substitutions and semantically homomorphic substitutions, arguing that logics must be closed under the former, but not (necessarily) under the latter. A substitution function from simple sentences into formulas of the object-language counts as semantically isomorphic "iff it preserves the semantical freedom of interpretations" ${ }^{4}$ it is homomorphic otherwise (cf. 2001: 371). A substitution $S$ preserves the semantical freedom of interpretations iff for any primitive $\varphi, s(\varphi)$ is such that if $\varphi$ is a logically contingent sentence with a certain degree of semantical freedom, then so is $s(\varphi)$. More technically, if $\langle\mathbf{W}, @, R, D\rangle$ is a Carnap-style constant domains model, the substitution function $s$ is said to be semantically isomorphic iff $\mathbf{W}_{s}=\mathbf{W}$, where $\mathbf{W}_{s}$ is the set of all sub-models for $s(\varphi)$, i.e. the set of all interpretations of sentences assigned by function $s$ to primitives. ${ }^{5}$ Schurz argued that closure under semantically isomorphic substitutions is "the strongest reasonable requirement concerning closure under substitutions" (2001: 371). Although Carnap-style modal semantics is not closed under semantically homomorphic substitutions, yet it is under semantically isomorphic substitutions, and that would be enough to ensure formality.

According to Schurz, all true sentences of the form $M \varphi$ are formal logical truths. Can Schurz's semantically isomorphic substitutivity replace US? Reasonably, it cannot without renouncing to define logical truths formally. To paraphrase Quine (1970: 50), Schurz's proposal to define logical truths in terms of formality should be something like: a logical truth is definable as a sentence from which we get only truths when we isomorphically substitute sentences for its simple sentences. Yet, since the notion of semantically isomorphic substitution is defined in terms of semantic modal notions, such as semantical freedom, logical possibility, logical contingency etc., logical truth cannot be formally captured in terms of semantically isomorphic substitutivity without circularity. ${ }^{6}$

${ }^{4}$ In informal words, by "semantical freedom of interpretations" with respect to a primitive sentence $\varphi$ Schurz means the space of all possible variations of interpretation of $\varphi$. For example, a substitution function $s$ from $\varphi$ to $\varphi \wedge \neg \varphi$ produces a decrease in the semantical freedom of $\varphi$ in that $\varphi$ can be true or false, while $\varphi \wedge \neg \varphi$ can only be false.

${ }^{5}$ For more details see Schurz (2001: 371 et seq.)

${ }^{6}$ Before speaking about semantically isomorphic substitutions, the author introduces syntactically isomorphic substitutions, which "replace primitives [...] by other primitives in a unique way" (Schurz 2001: 370). Syntactically isomorphic substitu- 


\subsection{McKeon's argument from analogy}

In this Section I will argue against what I have called McKeon's argument from analogy, according to which the notion of logical modality can be accommodated into Kripke semantics in virtue of an analogy between how first-order quantifiers and modal operators semantically work. Indeed, possible worlds semanticists usually interpret modal operators as quantifiers on possible worlds.

As we have seen in Section 2, first-order logical truths are said to be formal only if they are not context-sensitive. First-order logical truths context-insensitiveness entails their domain-insensitiveness: for any first-order sentence $\varphi$, for any $\psi$ obtained from $\varphi$ by (arbitrarily) restricting the quantifiers occurring in $\varphi$, if $\varphi$ is logically true, so is $\psi$. The property of being context-insensitive can be considered in one of its consequences, that is: if $\varphi$ is a first-order logical truth, $\varphi$ is compatible, so to speak, with all possible domains of discourse.

According to McKeon, such property has a straightforward transposition within modal logics, in virtue of the analogy between how firstorder quantifiers and modal operators work: just as first-order logical truths are such for any domain of discourse, so modal logical truths should be such for any logical space of logical possibilities, that is, for any set of logical possible worlds modal operators are restricted to. McKeon's argument from analogy rests on these premises. I will not proceed by questioning them, but rather by considering some of their consequences within a modal system representing logical modalities.

First-order domain-insensitiveness entails that for any first-order sentence $\varphi$, if $\varphi$ is logically true, then the sentence of the form $\varphi \wedge \exists \boldsymbol{x} \forall \boldsymbol{y}(\boldsymbol{x}=\boldsymbol{y})$ will be logically possible. That sentence says that the following conjunction is true: that $\varphi$ (e.g. "John passes the exam or John does not pass the exam"), and that there is exactly one object. If it were not, the sentence of the form $\neg \exists \boldsymbol{x} \forall \boldsymbol{y}(\boldsymbol{x}=\boldsymbol{y})$, or equivalently $\forall \boldsymbol{x} \exists \boldsymbol{y}(\boldsymbol{x} \neq \boldsymbol{y})$, which expresses the proposition that there are at least two things, would be a logical consequence of $\varphi$, whose truth would depend on the contingent fact that there is more than one thing. Accordingly, it comes to be essential to the logic of logical modality to assume the following meta-theorem: for any first-order sentence $\varphi$, if $L \varphi$ is true, so is $M(\varphi \wedge \exists \boldsymbol{x} \forall \boldsymbol{y}(\boldsymbol{x}=\boldsymbol{y}))$. At this point CI will require that the logical scheme $L \varphi \rightarrow M(\varphi \wedge \exists \boldsymbol{x} \forall \boldsymbol{y}(\boldsymbol{x}=\boldsymbol{y}))$ be valid for any first-order sentence $\varphi$, which is not the case in Kripke semantics under the logical reading of modal operators.

In fact, let $\varphi$ be a first-order logical truth. Let $\langle W, @, R, V\rangle$ be a Kripke model $\Re$ with the following characteristics: $W=\{@\}, d_{i}(@)=\left\{o_{1}, o_{2}\right\} . L \varphi$ will be true in $\mathscr{N}$, but $M(\varphi \wedge \exists \boldsymbol{x} \forall \boldsymbol{y}(\boldsymbol{x}=\boldsymbol{y}))$ will not, and that means: at

tions are defined purely syntactically, but they are only a proper subclass of semantically isomorphic substitutions. Thus what closure under syntactically isomorphic substitutions can at most do is to provide necessary but not sufficient conditions for the notion of logical truth. 
the logically possible state of affairs represented by @, the first-order logical truth $\varphi$ requires for its truth a domain of discourse consisting of more than one object.

We can conclude that McKeon's argument from analogy fails because it does not take into account the consequences of formalizing in the object-language (of the selected modal system) the first-order logical truths property of being context-insensitive, which is required by CI.

\section{Conclusion}

The formalization of logical modality in modal logical systems raises some serious problems for the formality of logic, when logical formality is syntactically understood in terms of uniform substitutivity. On the one hand, Carnap-style semantics violates the rule of uniform substitution. Schurz's proposal to redefine logical truth in terms of semantically isomorphic substitutions seems to be inappropriate, because of its circularity. On the other hand, Kripke semantics for the system $S_{5}$ is afflicted by inconsistency: in violation of what we have called "Condition of internalization" (CI), $\mathbf{S}_{5}$ is valid, but NLP is not a meta-theorem of $S_{5}$, since for any consistent but not valid first-order sentence $\varphi$, there is a counter Kripke model for $M \varphi$. In this paper, some reasons have been provided to extend the criticism of Kripke semantics for the system $S_{5}$ to every normal modal system aiming to represent logical modality. Such a system should contain both $\mathbf{S}_{4}$ (i.e. the internalization of $\mathrm{N}$ ) and $\mathbf{U S}$ (i.e. the internalization of US) as valid, but they are jointly inconsistent.

\section{References}

Beth, E. W. 1960. "Extension and Intension.” Synthese 12 (4): 375-379.

Carnap, R. 1947. Meaning and Necessity, 4th edition. Chicago: The University of Chicago Press.

Cocchiarella, N. 1975. "On the primary and secondary semantics of logical necessity." Journal of Philosophical Logic 4: 313-317.

Hanson, W. H. and J. Hawthorne 1985. "Validity in Intensional Languages." Notre Dame Journal of Formal Logic 26 (1): 9-35.

Kripke, S. 1959. "A Completeness Theorem in Modal Logic.” The Journal of Symbolic Logic 24 (1): 1-14.

Kripke, S. 1963. "Semantical considerations on modal logic." Acta Philosophica Fennica 16: 1-14.

Makinson, D. 1966. "How meaningful are modal operators." Australasian Journal of Philosophy 44 (3): 331-337.

McKeon, M. 2005. "A defense of the Kripkean account of logical truth in first-order modal logic." Journal of Philosophical Logic 34: 331-337.

Montague, R. 1974. "Logical Necessity, Physical Necessity, Ethics, and Quantifiers.” IR. n Thomason (ed.). Formal Philosophy. New Haven and London: Yale University Press: 71-83.

Pollock, J.L. 1966. "Model Theory and Modal Logic.” Logique et Analyse 9: 313-317. 
Quine, W. V. O. 1970. Philosophy of Logic. Harvard: Harvard University Press.

Schurz, G. 2001. “Carnap's Modal Logic.” In W. Stelzner and M. Stöckler (eds.). Zwischen traditioneller und moderner Logik. Nichtklassische Ansätze. Paderborn: Mentis: 365-380 